Research Article

\title{
Mathematical Analysis of Heat Transfer in Peristaltic Transport through a Rough Nonuniform Inclined Channel
}

\author{
R. Shukla ${ }^{D},{ }^{1}$ A. Medhavi, ${ }^{1}$ S. S. Bhatt ${ }^{(D)},{ }^{2}$ and R. Kumar ${ }^{3}$ \\ ${ }^{1}$ Department of Mechanical Engineering, Kamla Nehru Institute of Technology, Sultanpur, India \\ ${ }^{2}$ Department of Applied Sciences and Humanities, Faculty of Engineering \& Technology, Lucknow University, Lucknow, India \\ ${ }^{3}$ B. N. College of Engineering and Technology, Lucknow, India
}

Correspondence should be addressed to S. S. Bhatt; shankarbhatt56@gmail.com

Received 14 July 2020; Revised 4 August 2020; Accepted 8 August 2020; Published 24 August 2020

Guest Editor: Hussein Abulkasim

Copyright $(92020$ R. Shukla et al. This is an open access article distributed under the Creative Commons Attribution License, which permits unrestricted use, distribution, and reproduction in any medium, provided the original work is properly cited.

\begin{abstract}
A mathematical study for investigating heat transfer for the peristaltic flow of a Newtonian fluid through a nonuniform inclined channel while considering surface roughness of the inner wall has been conducted. Special attention is focused to analyze the physical behaviour of various flow characteristics at different magnitudes of surface roughness parameter. The results obtained for temperature have been characterized by two boundary conditions: uniform surface temperature and uniform heat flux. Effect of inner wall surface roughness and angle of inclination on the heat transfer for the peristaltic flow through a nonuniform channel has been discussed in detail. The expression for pressure gradient, velocity, and the temperature was obtained. The effect of inner wall surface roughness and the angle of inclination, on pressure rise and temperature, has also been elaborated in detail. The problem formulation developed has been solved using the long wavelength and low Reynolds number assumption.
\end{abstract}

\section{Introduction}

Peristaltic transport has gained the attention of researchers during the last five decades due to its applications in industry and physiology. Peristaltic motion involves a continuous progressive wave of area shrinking and enlargement, along the length of an expandable duct or tube. Peristaltic motion is an involuntary neuromuscular function, which occurs in several body organs of living beings, like the movement of fodder in the esophagus, chyme transport, urine deliver to the bladder through the ureter, transport mechanism in the cervical canal, ova transportation from the ovary to the uterus, fluid flow in ductus efferent, blood movement from small blood arteries, and lymph transportation through the lymphatic vessels. The principle involved in this type of motion is used for designing various contemporary medical equipments such as roller-finger pumps and pumping of blood in the heartlung machine. This mechanism is applied to the transport of toxic liquids in nuclear installations to prevent the mixing of radioactive elements in the environment. Apart from the above, this phenomenon finds applications in slurry transport, corrosive chemicals, and sanitary liquids from one point to the other.

Profound knowledge of the subject of peristaltic transport can be obtained from the vast literature available on the same. The first study in this area was conducted by Latham [1], which was further elaborated by Shapiro et al. [2] in detail. An effect of nonuniformity of the tube as well as the channel was observed in detail by Gupta and Seshadri [3] taking vas deferens as a reference. Heat transfer analysis of a toxic liquid subjected to peristaltic transport through a channel in nuclear operating power plants was carried out by Radhakrishnamacharya and Murty [4]. Excellent investigations have been done by El Koumy et al. [5] and Mekheimer et al. [6] to study the effect of constant magnetic field on peristaltic transport of electrically conducting Maxwell fluid, through a two-dimensional channel and microchannel, having porosity, with the consideration of Hall effect. An 
important study to find the effect of rotation on couple stress fluid flowing through a channel by peristaltic mechanism was done by Abd Elmaboud et al. [7]. Tripathi and Anwar Bég [8] studied peristaltic transport (chyme dynamics) through a diseased intestine which acts as a nonuniform porous medium. Two eminent investigations, for compressible, electrically conducting Jeffrey fluid and non-Newtonian nanofluid in a microfluidic and nonuniform channel considering the combined influence of the magnetic field and rheological properties with the consideration of slip velocity were conducted by Abdelsalam and Vafai [9] and Abdelsalam and Bhatti [10]. The collective impact of magnetic field, heat transfer, and porosity on the magneto-hydrodynamic peristaltic motion of the fluid through a channel has been investigated by Eldesoky et al. [11], and a decrement in temperature profile was found due to inclusion of fluid suspension. Victor and Shah [12, 13] found a solution for the energy equation of blood flow in a tube that is subjected to uniform wall temperature and heat flux in a fully developed region of velocity and temperature. The peristaltic mechanism exists in several body organs. The complications of blood flow using the peristaltic flow mechanism in a uniform and nonuniform channel as well as in tube were analyzed by Srivastava and Srivastava [14], supposing blood as a Casson fluid. Mekheimer [15] performed an analysis of blood flow during peristaltic motion through a nonuniform channel subjected to a magnetic field. An outstanding observation in the literature [16] is done to find the behavioural change in a small blood artery in which fluid with the suspended particle is transported by the peristaltic mechanism. Later, Abd Elmaboud et al. [17] conducted a study for the electromagnetic flow of two immiscible fluids, one permeable matrix soaked in a Newtonian fluid and the other pure fluid passes through the inclined channel, and it was found that electric field enhances velocity profile. Admirable investigations were done in studies by Abdelsalam et al. [18], Sohail et al. [19], and Abdelsalam and Sohail [20] to find the impact of heat and mass transfer during the transportation in the effect of the magnetic field through a two-dimensional channel and 3D flow. Sohail et al. [21] addressed the effect of momentum, heat, and mass transfer on the mixed convection boundary layer of Casson liquid for a porous medium. Sadaf and Abdelsalam [22] revealed the hostile effect of nanofluid in a wavy nonuniform annulus with flexible boundaries. Some other worthy research studies by Abdelsalam and Bhatti [23], Abdelsalam and Bhatti [24], Abdelsalam and Bhatti [25], and Eldesoky et al. [26] have been performed with magnetic field effect in a tube for blood flow. Biological organs in which biofluids flow have a small degree of roughness or uncertain (random) spatial variability. Park et al. [27] calculated the effective blood viscosity, with the observation of endothelium roughness of coronary arteries. The degree of roughness within the tissues is very helpful in recognizing the diseases. Evaluation of surface roughness of coronary arteries, in which blood flow takes place through the peristaltic mechanism, helps in developing a remedy for coronary heart disease. Burton and Espino [28] investigated the consequences of intense physiological loading on surface roughness; from the observations, it was revealed that initially, mechanical overloading has no significant effect on roughness; however, when a chemical processing was applied on the surface, an increase in surface roughness was noticed in the longitudinal and circumferential directions. This intense overloading results in damage to the arteries, which is notable after dehydration of the tissue.

Several analyses have been performed in the present area for mini and microchannels. Bhatti et al. [29] conducted a study on the swimming pattern of gyrotactic microorganisms in a blood-based nanofluid flowing through the anisotropically narrowing artery. Veatch [30] carried out experiments on eleven rectangular passages of different cross sections, sizes, and roughness; experiments were performed using four different fluids, and it was concluded that, for laminar flow, friction factor and Reynolds number are independent of roughness. Wall roughness has a significant impact on the heat transfer rate during fluid motion. Study of the impact of wall roughness while considering the roughness orientation, roughness structure, and shape of the roughness element has become a key area of research due to its vast applications in engineering. Excellent studies in the field of heat transfer for the roughed wall have been performed by Han et al. [31] and Taslim et al. [32]. Taylor et al. [33] found the influence of roughness on the fluid motion for surfaces having up to $14 \%$ relative roughness, and constricted flow diameters were not considered for analysis during the study; however, till that time, the studies were limited to $5 \%$ of relative roughness, which violated micro- and macrofluidic flow systems. Wagner and Kandlikar [34] and Dharaiya and Kandlikar [35] investigated experimentally the influence of sinusoidal structured roughness on heat transfer rate during liquid transport through micro- and macrochannels; experiments were performed for different pitches and roughness heights for a correct understanding of frictional losses in fully developed laminar flow. Kharati-Koopaee and Zare [36] and Attalla et al. [37] performed a study to find the heat transfer effect during fluid flow in microchannels having a rectangular cross section with aligned and offset structured sinusoidal roughness. From 
the above study, it was concluded that, to lower the pressure loss for the fluid under consideration, high roughness height or low channel height gave favourable results.

From the above, it is evident that, though many investigations have been undertaken for explaining the variation in heat transfer rate due to surface roughness during fluid flow, the variation in heat transfer rate due to wall roughness for peristalsis mechanism during transport of fluid has yet to be explored. The current analysis involves the study of heat transfer, while pondering the wall roughness for a two-dimensional nonuniform inclined channel when a Newtonian fluid flows through it under the influence of peristaltic waves.

\section{Problem Formulation}

Figure 1 shows the flow geometry, for the laboratory frame of reference. It is supposed that the walls of the nonuniform channel have sinusoidal-shaped roughness. The study has been carried out in the rectangular coordinate system $(X, Y)$.

The wall geometry is

$$
H(X, t)=d(X)+b \sin \left(\frac{(2 \pi(X-c t))}{\lambda}\right)-b_{1} \cos ^{4}\left(\frac{\pi X}{\lambda_{1}}\right),
$$

with

$$
d(X)=a+K X,
$$

where $a$ represents the half channel width at entry, $b$ is the amplitude, $\lambda$ is the wavelength, $c$ is the wave propagation velocity, $K$ is the nonuniformity parameter, $\lambda_{1}$ is the pitch, $t$ is the time, $X$ is the axial variable, and $b_{1}$ is the roughness height.

Continuity equation is as follows:

$$
\frac{\partial U}{\partial X}+\frac{\partial U}{\partial Y}=0
$$

Momentum equation is as follows:

$$
\begin{aligned}
& \rho\left(\frac{\partial U}{\partial t}+U \frac{\partial U}{\partial X}+V \frac{\partial U}{\partial Y}\right)=-\frac{\partial p}{\partial X}+\mu\left(\frac{\partial^{2} U}{\partial X^{2}}+\frac{\partial^{2} U}{\partial Y^{2}}\right)+\rho g \sin \alpha \\
& \rho\left(\frac{\partial V}{\partial t}+U \frac{\partial V}{\partial X}+V \frac{\partial V}{\partial Y}\right)=-\frac{\partial p}{\partial Y}+\mu\left(\frac{\partial^{2} V}{\partial X^{2}}+\frac{\partial^{2} V}{\partial Y^{2}}\right)-\rho g \cos \alpha
\end{aligned}
$$

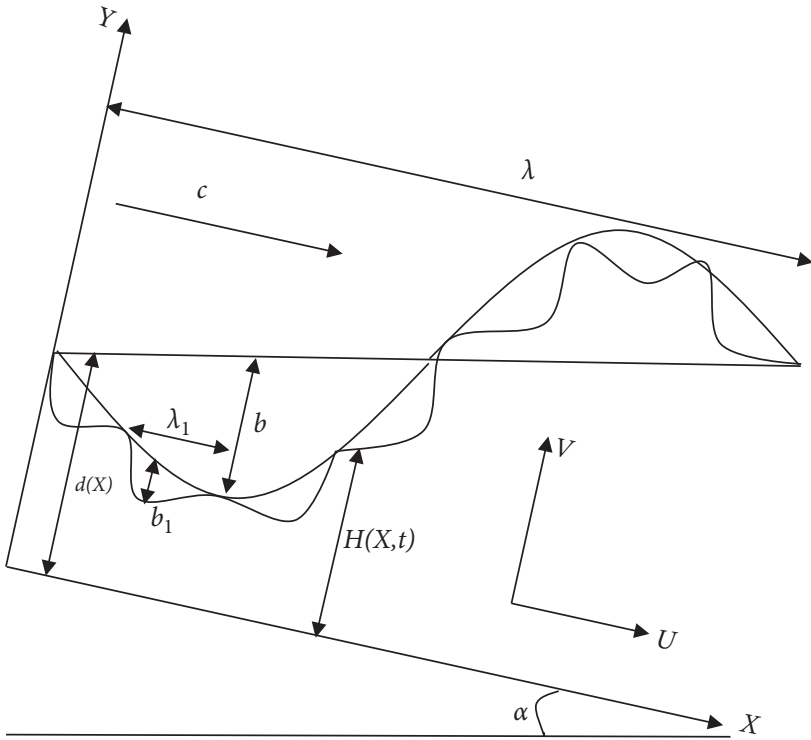

Figure 1: Geometry of the problem.

$$
\begin{aligned}
C_{p}\left(\frac{\partial T}{\partial t}+U \frac{\partial T}{\partial X}+V \frac{\partial T}{\partial Y}\right)= & \frac{k}{\rho}\left(\frac{\partial^{2} T}{\partial X^{2}}+\frac{\partial^{2} T}{\partial Y^{2}}\right)+\nu\left(2 \left(\left(\frac{\partial U}{\partial X}\right)^{2}\right.\right. \\
& \left.\left.+\left(\frac{\partial V}{\partial Y}\right)^{2}\right)+\left(\frac{\partial U}{\partial X}+\frac{\partial V}{\partial Y}\right)^{2}\right)
\end{aligned}
$$

with boundary conditions

$$
\begin{cases}U=0, & \text { at } Y=H, \\ \frac{\partial U}{\partial Y}=0, & \text { at } Y=0, \\ T=T_{c}, & \text { at } Y=0, \\ T=T_{w}, & \text { at } Y=H,\end{cases}
$$

where $U$ and $V$ are the velocity components for $X$ and $Y$ directions, respectively, in the fixed frame of reference, $p$ is the pressure, $\rho$ is the density, $\vartheta$ is the kinematic coefficient of viscosity, $c_{p}$ is the specific heat at constant pressure, $T$ is the temperature of fluid, $k$ is the thermal conductivity, $T_{c}$ is the temperature at the centre of channel, $T_{w}$ is the temperature at the wall, and $\alpha$ is the angle of inclination. 


\section{Analysis}

The nondimensional parameters are as follows:

$$
\begin{aligned}
& U^{\prime}=\frac{U}{c}, \\
& V^{\prime}=\frac{V}{c \delta}, \\
& X^{\prime}=\frac{X}{\lambda}, \\
& Y^{\prime}=\frac{Y}{a}, \\
& \theta_{H}=\frac{T-T_{c}}{T_{w}-T_{c}}, \\
& \theta_{T}=\frac{T-T_{w}}{T_{c}-T_{w}}, \\
& t^{\prime}=\frac{c t}{\lambda} \text {, } \\
& p^{\prime}=\frac{a^{2}}{c \mu \lambda} p, \\
& \delta=\frac{a}{\lambda}, \\
& \phi=\frac{b}{a}, \\
& \operatorname{Pr}=\frac{\mu C_{p}}{k}, \\
& \mathrm{Ec}_{H}=\frac{c^{2}}{\left(T_{w}-T_{c}\right) C_{p}}, \\
& \phi_{1}=\frac{b_{1}}{a} \text {, } \\
& \operatorname{Re}=\frac{\rho a c}{\mu}, \\
& \lambda_{1}^{\prime}=\frac{\lambda_{1}}{\lambda}, \\
& \mathrm{Ec}_{T}=\frac{c^{2}}{\left(T_{c}-T_{w}\right) C_{p}}, \\
& \mathrm{Fr}=\frac{c^{2}}{g a} \text {, }
\end{aligned}
$$

where Re is the Reynolds Number, $\delta$ is the wavenumber, $\theta_{H}$ is the dimensionless temperature in the case of uniform heat flux, $\theta_{T}$ is the dimensionless temperature in the case of uniform surface temperature, $\mathrm{Ec}_{H}$ is the Eckert number in the case of uniform heat flux, $\mathrm{Ec}_{T}$ is the Eckert number in the case of uniform surface temperature, and $\operatorname{Pr}$ is the Prandtl number.

Using equation (8) in equations (3)-(6) and dropping primes with the supposition of long wavelength and low Reynolds number,

$$
\begin{aligned}
\frac{\partial U}{\partial X}+\frac{\partial U}{\partial Y} & =0 \\
\frac{\partial^{2} U}{\partial Y^{2}}+\frac{\operatorname{Re}}{\operatorname{Fr}} \sin \alpha & =\frac{\partial p}{\partial X}, \\
\frac{\partial p}{\partial Y} & =0 \\
\frac{\partial^{2} \theta}{\partial Y^{2}}+\operatorname{Ec} \operatorname{Pr}\left(\frac{\partial U}{\partial Y}\right)^{2} & =0
\end{aligned}
$$

with boundary conditions

$$
\begin{cases}U=0, & \text { at } Y=h=\frac{H}{a}=1+\frac{K \lambda X}{a}+\phi \sin (2 \pi(X-t))-\phi_{1} \cos ^{4}\left(\frac{\pi X}{\lambda_{1}}\right), \\ \frac{\partial U}{\partial Y}=0, & \text { at } Y=0, \\ \theta_{H}=0, \theta_{T}=1, & \text { at } Y=0, \\ \theta_{H}=1, \theta_{T}=0, & \text { at } Y=h,\end{cases}
$$


where $K$ is the nonuniformity of the wall, $\phi$ is the amplitude ratio, $\phi_{1}$ is the roughness parameter, and $\lambda_{1}$ is the dimensionless pitch.

On solving equations (9)-(12) with given boundary conditions equation (13), we get

$$
\begin{aligned}
U & =\left(\frac{Y^{2}-h^{2}}{2}\right)\left(\frac{\mathrm{d} p}{\mathrm{~d} X}-\frac{\mathrm{Re}}{\mathrm{Fr}} \sin \alpha\right), \\
\theta_{H} & =\mathrm{Ec}_{H} \operatorname{Pr}\left(\frac{\mathrm{d} p}{\mathrm{~d} X}-\frac{\operatorname{Re}}{\mathrm{Fr}} \sin \alpha\right)^{2}\left(\frac{h^{3} y-y^{4}}{12}\right)+\frac{y}{h}, \\
\theta_{T} & =\mathrm{Ec}_{T} \operatorname{Pr}\left(\frac{\mathrm{d} p}{\mathrm{~d} X}-\frac{\operatorname{Re}}{\mathrm{Fr}} \sin \alpha\right)^{2}\left(\frac{h^{3} y-y^{4}}{12}\right)-\frac{y}{h}+1 .
\end{aligned}
$$

The flow flux $q(X, t)$ is found as

$$
q(X, t)=\int_{0}^{h} U \mathrm{~d} Y=-\frac{h^{3}}{3}\left(\frac{\mathrm{d} p}{\mathrm{~d} X}-\frac{\mathrm{Re}}{\mathrm{Fr}} \sin \alpha\right)
$$

or

$$
\frac{\mathrm{d} p}{\mathrm{~d} X}=\frac{-3 q(X, t)}{h^{3}}+\frac{\mathrm{Re}}{\mathrm{Fr}} \sin \alpha .
$$

As the roughness parameter $\phi_{1} \longrightarrow 0$ and angle of inclination $\alpha \longrightarrow 0$, the result obtained from equation (16) is the same as that found by Gupta and Seshadri [3].

The pressure rise $\Delta p$ across the channel of $L$ length in dimensionless forms is

$$
\Delta p=\int_{0}^{L / \lambda}\left(\frac{\mathrm{d} p}{\mathrm{~d} X}\right) \mathrm{d} X
$$

\section{Results and Discussion}

In the present study, graphs have been drawn using MATHEMATICA software. The instantaneous flow rate $q(x$, $t)$, periodic in $(x-t)$, is as follows [3]:

$$
q(X, t)=Q+\phi \sin 2 \pi(X-t),
$$

and magnitude of various parameters is taken as $a=$ $0.01 \mathrm{~cm}, L=\lambda=10 \mathrm{~cm}, K=0.5(a / L)=0.0005[14,15]$, and $\phi_{1}=\left(b_{1} / a\right)$; the magnitude of $b_{1}$ is from [28].

Figure 2 depicts changes in the pressure rise as a function of time over one period of the wave, at different surface roughness parameters and channel inclinations, at a particular value of all other parameters like $K, Q, \lambda_{1}, \phi_{1}, \operatorname{Re}$, and Fr. It is obvious from Figure 2 that, during one period of the wave, an increase in the angle of inclination enhances the pressure rise. The effect of surface roughness is most significant at the time $t \approx 0.3$, which corresponds to the point where maximum occlusion occurs from the entry of the channel. It is seen that pressure rise increases with an increase in the roughness parameter for a particular choice of all other parameters. This can be interpreted that, with increasing surface roughness at any particular section, the cross section area will reduce due to which the pressure rise increases with increasing surface roughness at that section.

Figure 3 displays the changes in pressure rise against flow rate for various surface roughness parameters at different angles of inclinations, at the particular value of all other parameters $\left(K, t, \lambda_{1}, \phi, \mathrm{Re}\right.$, and $\left.\mathrm{Fr}\right)$. The magnitude of the pressure rise decreases linearly with an increase in flow rate for all magnitude of the surface roughness parameter and angle of inclination. In a noninclined channel, the maximum flow rate occurs up to $Q=0.64$, and increase in inclination from 0 to $(\pi / 2)$ enhances the flow rate. At any particular value of flow rate in-between $Q=0$ and 0.64 , pressure rise increases continuously; when the surface roughness parameter increases from $Q=0.64$ to $Q=1$, reverse impact on the value of pressure rise is seen with increasing surface roughness parameter. The pressure rise increases also with an increase in the angle of inclination from 0 to $(\pi / 2)$. The pressure rise is maximum at no flow condition, and this value rises with the surface roughness parameter and angle of inclination. It can be observed that, in the peristaltic pumping zone, the maximum flow rate is obtained at zero pressure rise. From Figure 3, it is also observed that the magnitude of the maximum flow rate can be enhanced by increasing the angle of inclination for a particular choice of all other parameters.

The amplitude ratio has a significant impact on the pressure rise. Figure 4 shows that, at higher magnitudes of amplitude ratio $(\phi>0.8)$, a rapid augment is visualised in the magnitude of pressure rise. With an increase in inclination from 0 to $(\pi / 2)$, the pressure rise increases at any particular value of amplitude ratio.

Figures 5 and 6 depict the variation in pressure rise against flow rate at different values of Re and Fr, respectively, for different surface roughness parameters. It can be noticed from Figures 5 and 6 that the pressure rise increases with an increase in surface roughness parameter at zero flow rate, and this difference goes on reducing as flow rate increases.

From Figure 5, it is also observed that, for different values of surface roughness parameter, the magnitude of maximum pressure rise and maximum flow rate increases with an increase in Re. Again from Figure 6, it is seen that the value of maximum pressure rise and maximum flow rate decreases with an increase in Fr for different values of surface roughness parameter. Variation in flow rate and pressure rise with Fr has opposite nature to that of Re.

Figures 7(a) and 7(b) and Table 1 describe the effect of the angle of inclination on the temperature distribution for different values of surface roughness parameter; similarly, Figures 8(a) and 8(b), Table 2, Figures 9(a) and 9(b), and Table 3 describe the effect of Re and Fr on the temperature distribution at different values of surface roughness parameter. The entire analysis performed above has been carried out under two conditions, uniform heat flux and uniform surface temperature condition.

Figures 7 (a) and 7(b) and Table 1 describe the effect of the angle of inclination on the temperature distribution for different values of surface roughness parameter; similarly, Figures 8(a) and 8(b), Table 2, Figures 9(a) and 9(b), and Table 3 describe the effect of Re and Fr on the temperature 


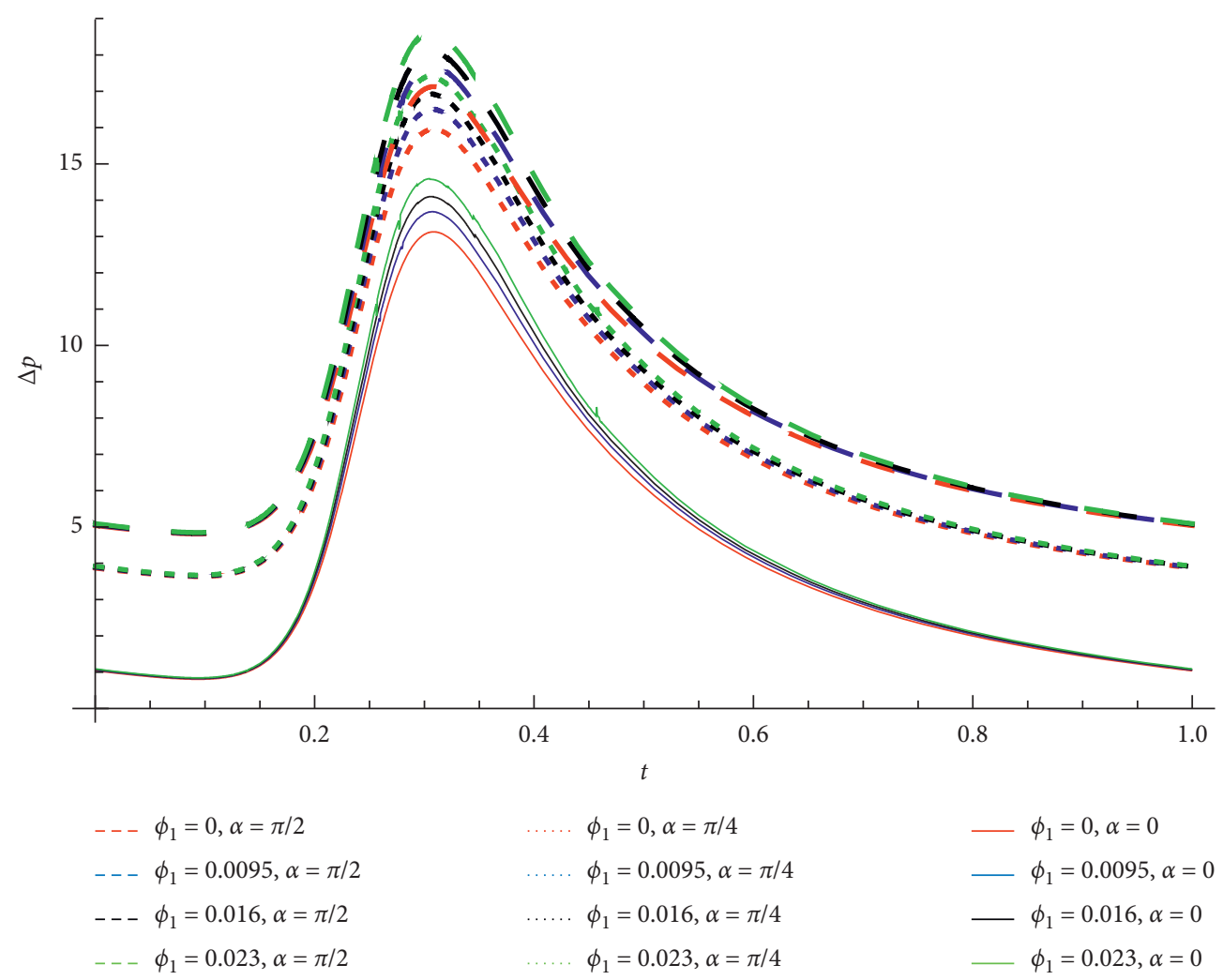

Figure 2: Pressure rise versus time for various values of $\phi_{1}, \alpha$ at $K=0.0005, Q=0.3, \lambda_{1}=0.00011, \phi=0.8, \operatorname{Re}=0.4$, and $\mathrm{Fr}=0.1$.

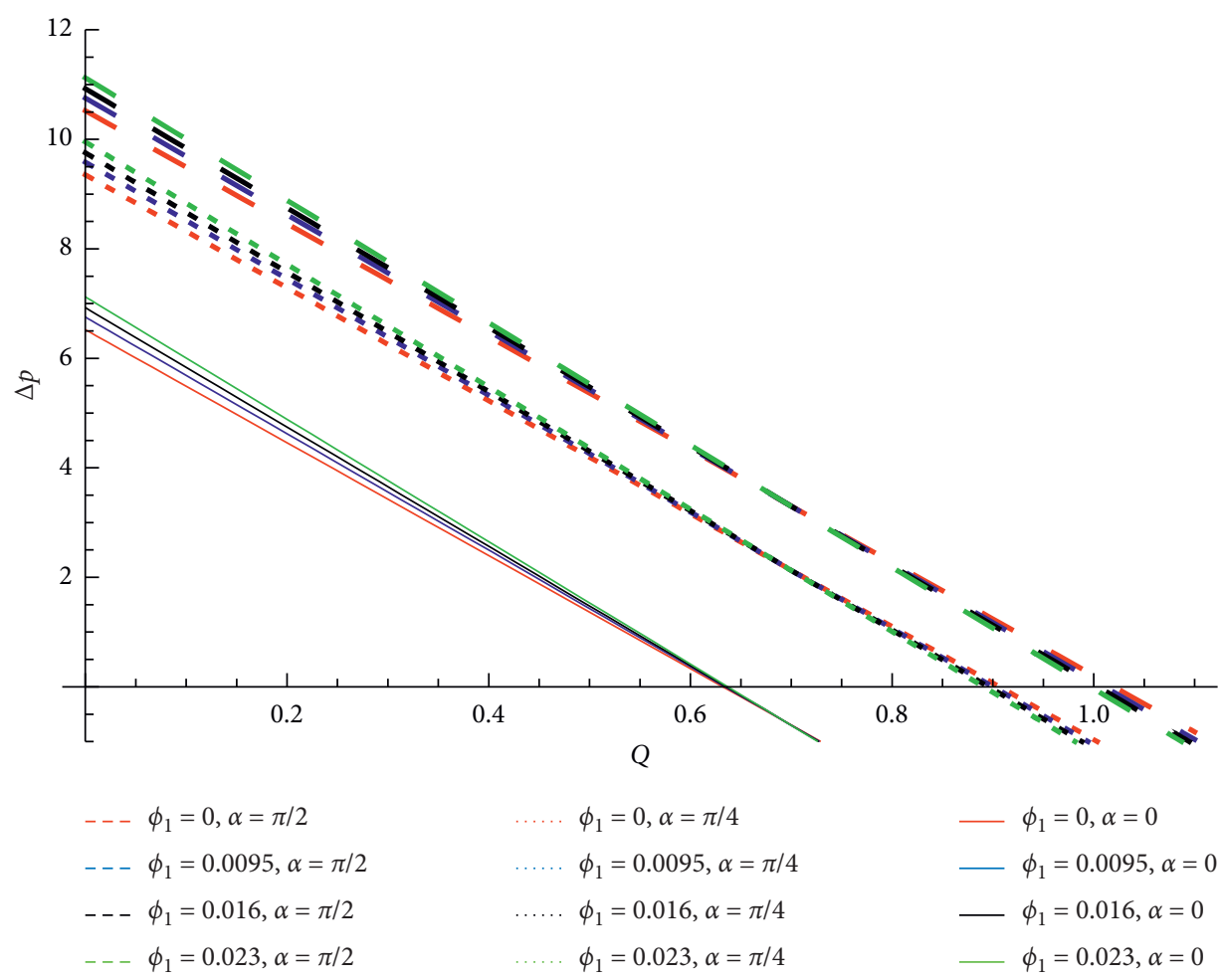

Figure 3: Pressure rise versus flow rate for different values of $\phi_{1}, \alpha$ at $K=0.0005, t=0.2, \lambda_{1}=0.00011, \phi=0.8, \operatorname{Re}=0.4$, and Fr $=0.1$. 


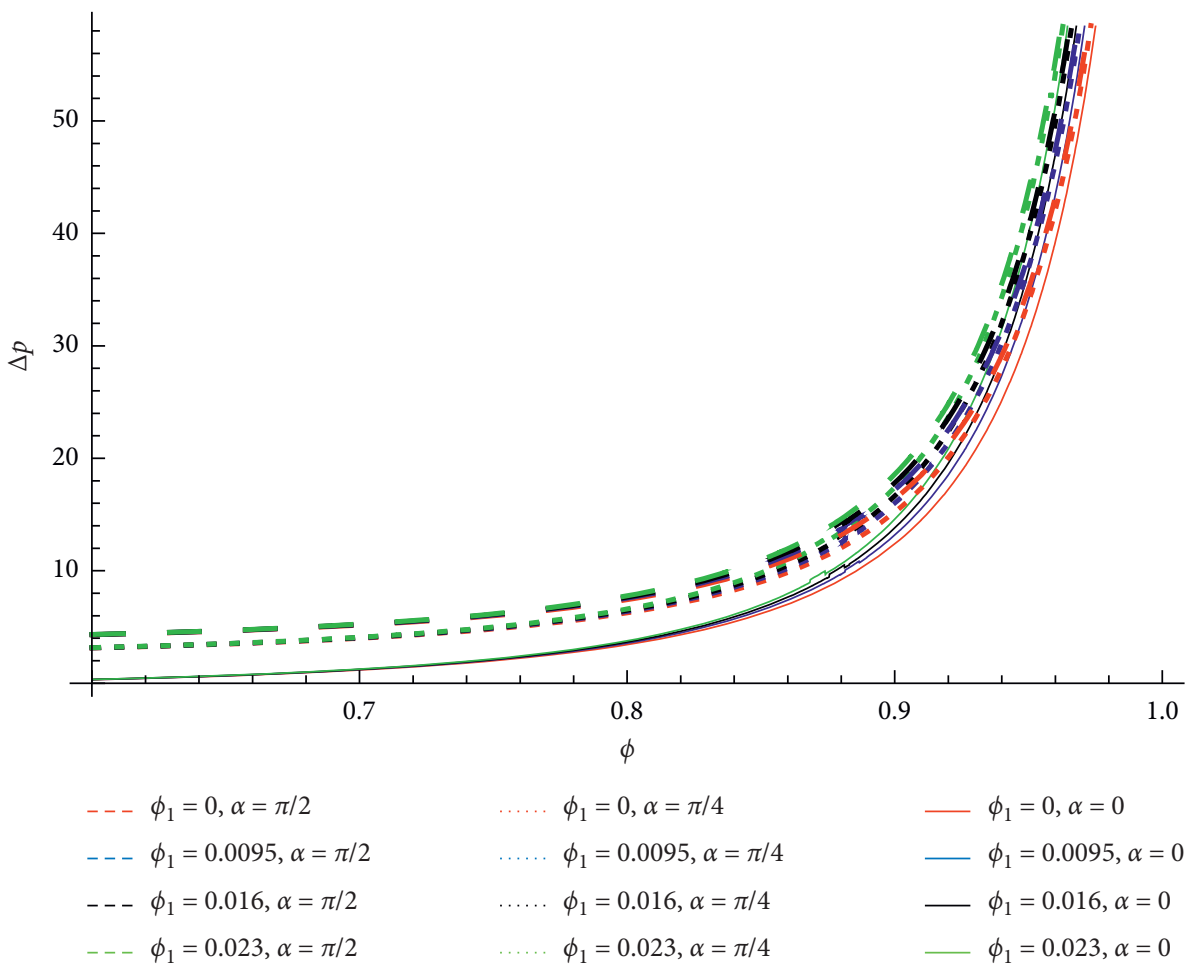

Figure 4: Pressure rise versus amplitude ratio for different values of $\phi_{1}, \alpha$ at $K=0.0005, t=0.2, \lambda_{1}=0.00011, Q=0.3, \operatorname{Re}=0.4$, and $\mathrm{Fr}=0.1$.

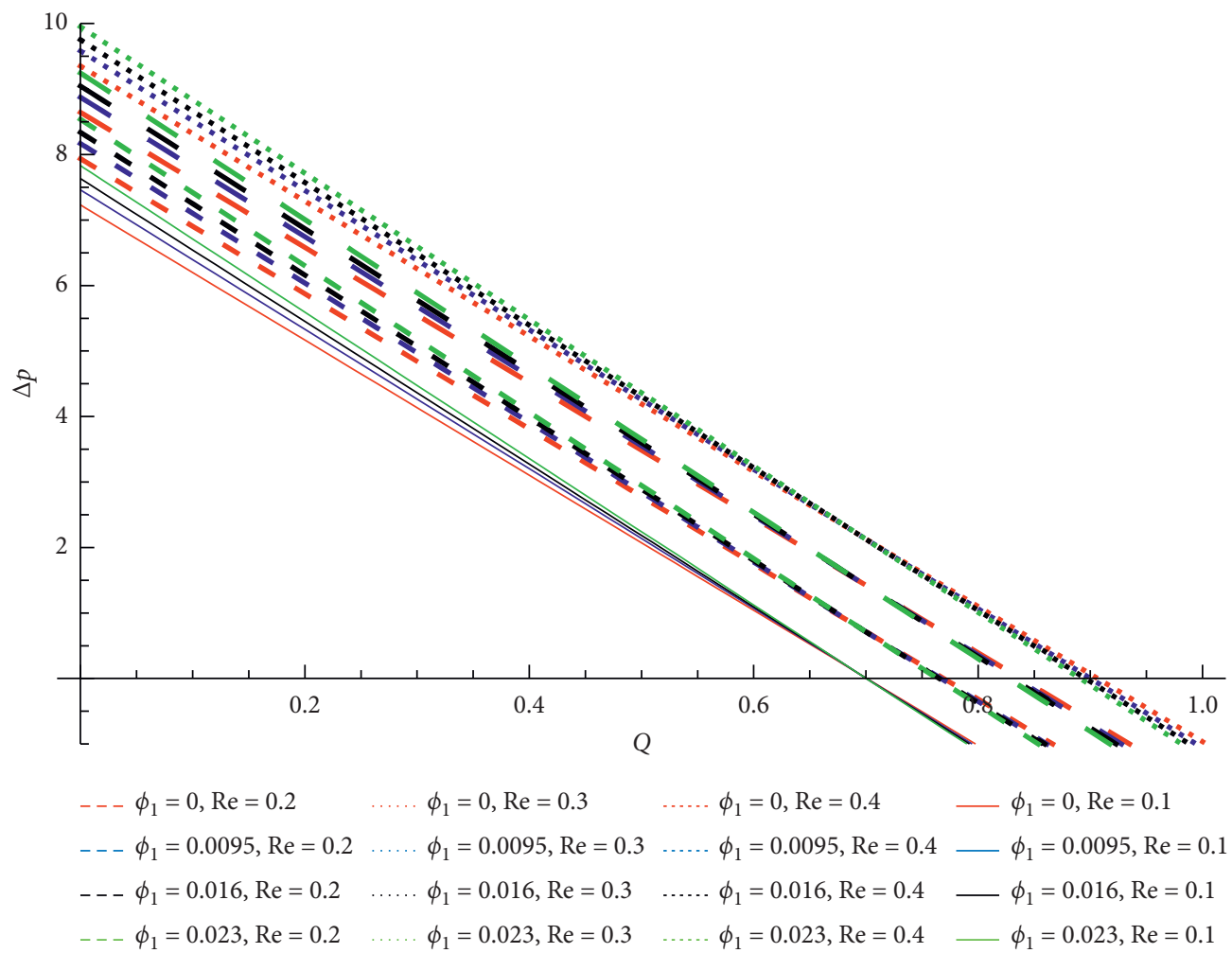

Figure 5: Pressure rise versus flow rate for different values of $\phi_{1}$, Re at $K=0.0005, t=0.2, \lambda_{1}=0.00011, \phi=0.8, \alpha=(\pi / 4)$, and Fr $=0.1$. 


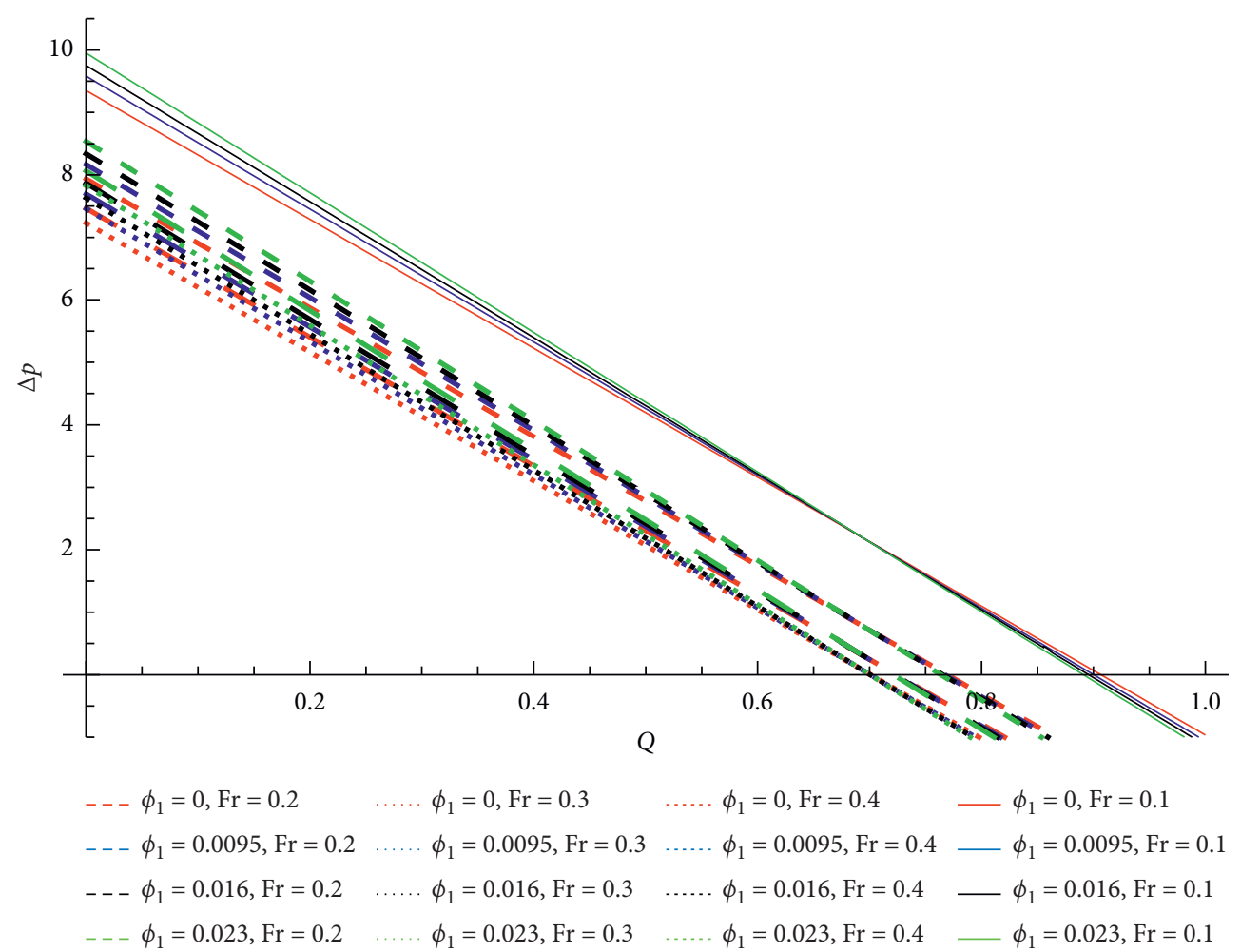

Figure 6: Pressure rise versus flow rate for different values of $\phi_{1}$, Fr at $K=0.0005, t=0.2, \lambda_{1}=0.00011, \phi=0.8, \alpha=(\pi / 4)$, and $\operatorname{Re}=0.4$.

distribution at different values of surface roughness parameter. The entire analysis performed above has been carried out under two conditions: uniform heat flux and uniform surface temperature condition.

Figure 7 (a) shows the variation in $\theta_{H}$ against $y$ for several values of channel inclination and surface roughness. As $y$ increases, $\theta_{H}$ increases from a minimum value at the centre of the channel to a maximum value at the channel wall, for a channel having zero inclination. For a noninclined channel, it is visible from Table 1 that $\theta_{H}$ increases and $\theta_{T}$ decreases at any particular value of $y$, as the surface roughness parameter is increased. It is also concluded from Table 1 that, for a particular value of surface roughness parameter in a noninclined channel, when $y$ increases, $\theta_{H}$ increases and $\theta_{T}$ decreases.

With the variation in $\theta_{H}$ against $y$, for channels inclined at $(\pi / 4)$ and $(\pi / 2)$ inclination, initially $\theta_{H}$ increases rapidly to a maximum value after which it starts decreasing; finally, it coincides with the maximum value of $\theta_{H}$ for a channel having zero inclination, at the wall of the channel. Furthermore, for channels inclined at $(\pi / 4)$ and $(\pi / 2)$, from Table 1 , it is found that the value $\theta_{H}$ and $\theta_{T}$ decreases at any particular value of $y$, as the value of surface roughness parameter increases. For any particular value of surface roughness, with an increase in $y, \theta_{H}$ increases and $\theta_{T}$ decreases for a channel having $(\pi / 4)$ inclination. It is also clear from Table 1 that, for any particular value of surface roughness, $\theta_{H}$ increases as $y$ increases from 0 to 0.8 , after which it starts decreasing and $\theta_{T}$ increases as $y$ increases from 0 to 0.4 , after which it starts decreasing for a channel having $(\pi / 2)$ inclination.
Figure $7(\mathrm{~b})$ shows the variation in $\theta_{T}$ against $y$ for different values of channel inclination and surface roughness. The value of $\theta_{T}$ decreases with increase in $y$ and at $y=0 ; \theta_{T}$ is maximum, for channels having zero and $(\pi / 4)$ inclination. But in the case of channels having $(\pi / 2)$ inclination, with increase in $y, \theta_{T}$ increases up to a maximum value, and then, it decreases to zero at the channel wall.

Figures $8(a)$ and $8(b)$ show the temperature profile for different values of Re and surface roughness parameters for both boundary conditions mentioned above, i.e., uniform heat flux and uniform surface temperature condition. The above is also described numerically through data presented in Table 2. Figures 9(a) and 9(b) show the temperature profile for different values of $\mathrm{Fr}$ and surface roughness parameters, and the same is described numerically through the data presented in Table 3. From the above two figures, it is clear that temperature variation at different values of Re and Fr follows a similar pattern to that depicted in Figures 7(a) and 7(b) except in the case of a channel having $(\pi / 2)$ inclination, wherein $\theta_{T}$ initially increases and then it decreases. The variation in $\theta_{T}$ for different values of Re and Fr is similar in nature; $\theta_{T}$ decreases with increasing $y$ for all values of Re and Fr. From Table 2, it is clear that, at $R e=0.1$ and 0.2 , for any particular value of $y, \theta_{H}$ goes on increasing, while $\theta_{T}$ goes on decreasing upon increasing surface roughness parameter. As the value of the surface roughness parameter is incremented, at $\operatorname{Re}=0.3$, for a particular value of $y, \theta_{H}$ decreases for all values of $y$ from 0 to 0.8 , and $\theta_{T}$ also decreases for all values of $y$. Excluding the value of $\theta_{H}$ at $y=0.4$, the variation in $\theta_{H}$ and $\theta_{T}$, for any particular value of $y$, with increasing values of surface roughness parameter 


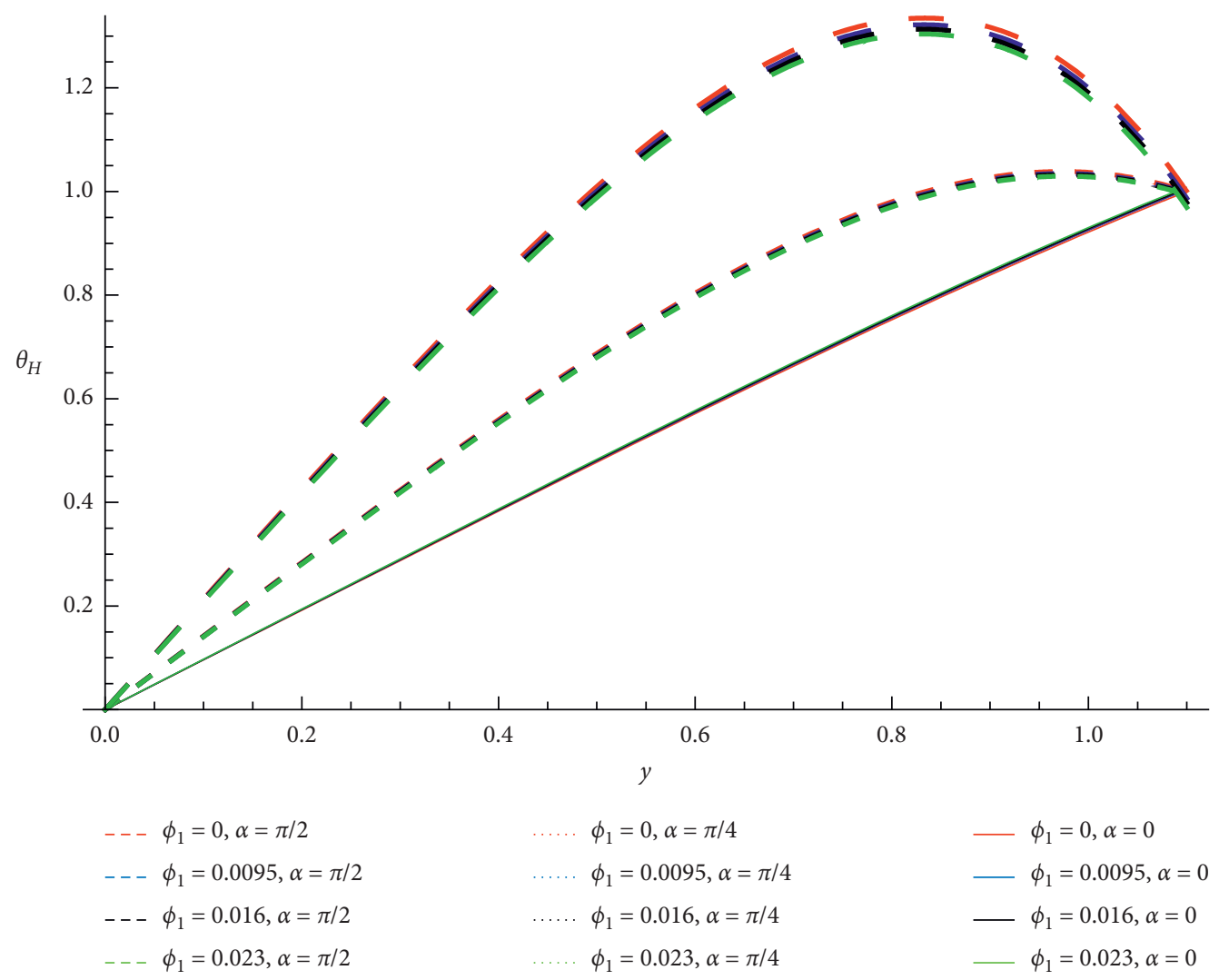

(a)

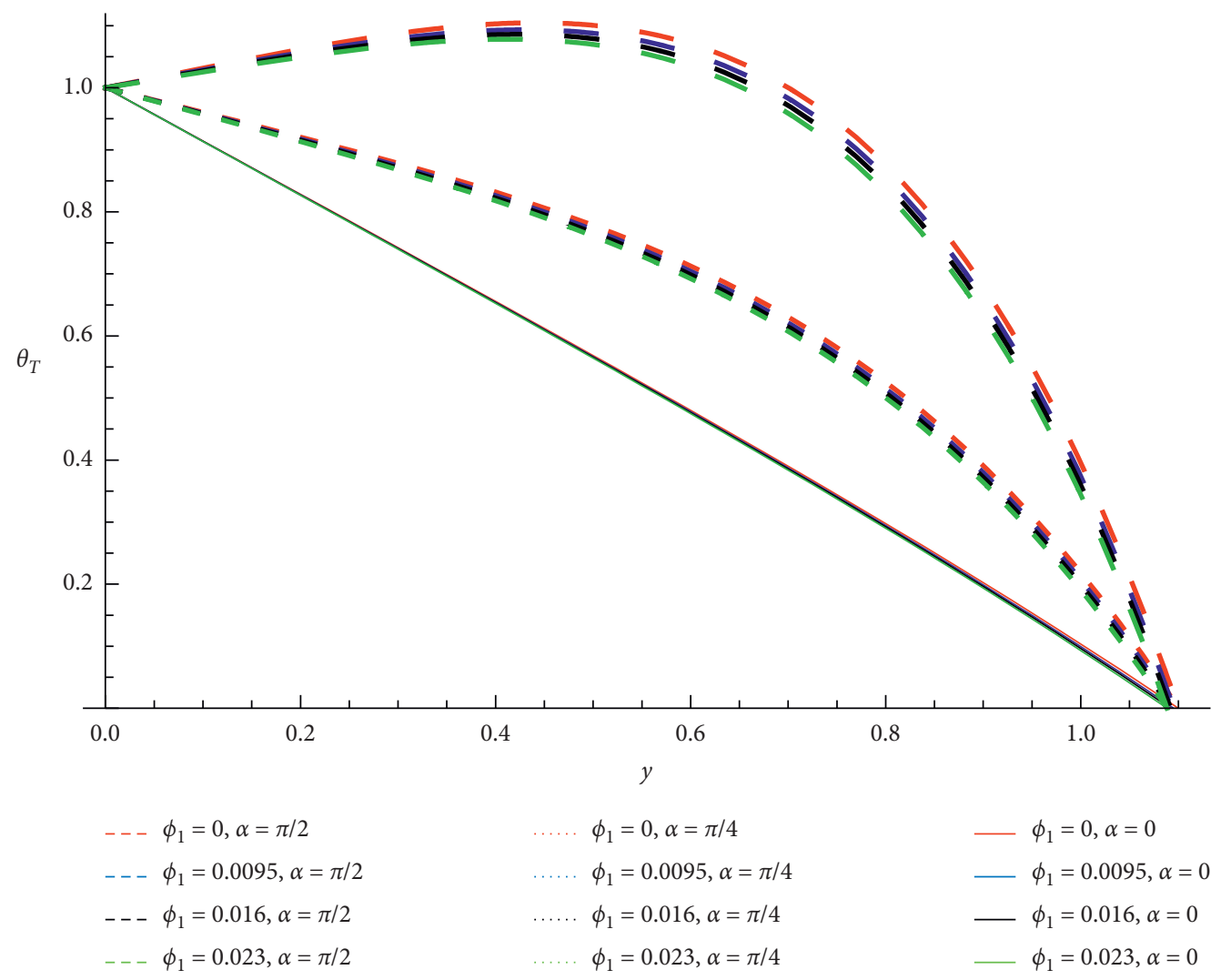

(b)

Figure 7: Temperature vs $y$ for different values of $\phi_{1}, \alpha$ at $K=0.0005, t=0.2, \lambda_{1}=0.00011, x=0.2, \phi=$ $0.8, \operatorname{Re}=0.4, \mathrm{Fr}=0.1, \mathrm{Q}=0.3, \operatorname{Pr}=1, \mathrm{Ec}_{H}=1$, and $\mathrm{Ec}_{T}=1$ : (a) uniform heat flux condition; (b) uniform surface temperature. 
TABLE 1: Temperature distribution at different values of angle of inclination and surface roughness parameter.

\begin{tabular}{|c|c|c|c|c|c|c|c|c|}
\hline \multirow[b]{2}{*}{$y$} & \multicolumn{4}{|c|}{$\begin{array}{c}\theta_{T} \\
\alpha=0\end{array}$} & \multicolumn{4}{|c|}{$\begin{array}{c}\theta_{H} \\
\alpha=0\end{array}$} \\
\hline & $\phi_{1}=0$ & $\phi_{1}=0.0095$ & $\phi_{1}=0.016$ & $\phi_{1}=0.023$ & $\phi_{1}=0$ & $\phi_{1}=0.0095$ & $\phi_{1}=0.016$ & $\phi_{1}=0.023$ \\
\hline 0 & 1 & 1 & 1 & 1 & 0 & 0 & 0 & 0 \\
\hline 0.2 & 0.8282 & 0.8276 & 0.8271 & 0.8266 & 0.1919 & 0.1928 & 0.1934 & 0.1941 \\
\hline 0.4 & 0.6556 & 0.6543 & 0.6534 & 0.6524 & 0.3829 & 0.3847 & 0.3860 & 0.3873 \\
\hline 0.6 & 0.4800 & 0.4779 & 0.4764 & 0.4749 & 0.5709 & 0.5735 & 0.5754 & 0.5773 \\
\hline 0.8 & 0.2976 & 0.2946 & 0.2925 & 0.2902 & 0.7522 & 0.7555 & 0.7577 & 0.7602 \\
\hline \multirow[t]{2}{*}{1.0} & 0.1035 & 0.0992 & 0.0962 & 0.0930 & 0.9217 & 0.9253 & 0.9277 & 0.9304 \\
\hline & \multicolumn{4}{|c|}{$\alpha=(\pi / 4)$} & \multicolumn{4}{|c|}{$\alpha=(\pi / 4)$} \\
\hline 0 & 1 & 1 & 1 & 1 & 0 & 0 & 0 & 0 \\
\hline 0.2 & 0.9203 & 0.9173 & 0.9153 & 0.9132 & 0.2839 & 0.2825 & 0.2816 & 0.2807 \\
\hline 0.4 & 0.8319 & 0.8261 & 0.8222 & 0.8179 & 0.5592 & 0.5566 & 0.5548 & 0.5529 \\
\hline 0.6 & 0.7127 & 0.7043 & 0.6986 & 0.6924 & 0.8036 & 0.7999 & 0.7975 & 0.7948 \\
\hline 0.8 & 0.5256 & 0.5151 & 0.5080 & 0.5003 & 0.9801 & 0.9760 & 0.9732 & 0.9702 \\
\hline \multirow[t]{2}{*}{1.0} & 0.2186 & 0.2070 & 0.1992 & 0.1908 & 1.0368 & 1.0331 & 1.0307 & 1.0282 \\
\hline & \multicolumn{4}{|c|}{$\alpha=(\pi / 2)$} & \multicolumn{4}{|c|}{$\alpha=(\pi / 2)$} \\
\hline 0 & 1 & 1 & 1 & 1 & 0 & 0 & 0 & 0 \\
\hline 0.2 & 1.0617 & 1.0565 & 1.0529 & 1.0491 & 0.4254 & 0.4217 & 0.4192 & 0.4166 \\
\hline 0.4 & 1.1029 & 1.0925 & 1.0855 & 1.0779 & 0.8302 & 0.8230 & 0.8181 & 0.8129 \\
\hline 0.6 & 1.0704 & 1.0553 & 1.0451 & 1.0341 & 1.1613 & 1.1510 & 1.1440 & 1.1365 \\
\hline 0.8 & 0.8759 & 0.8569 & 0.8440 & 0.8303 & 1.3304 & 1.3178 & 1.3093 & 1.3002 \\
\hline 1.0 & 0.3956 & 0.3743 & 0.3598 & 0.3444 & 1.2138 & 1.2004 & 1.1914 & 1.1818 \\
\hline
\end{tabular}

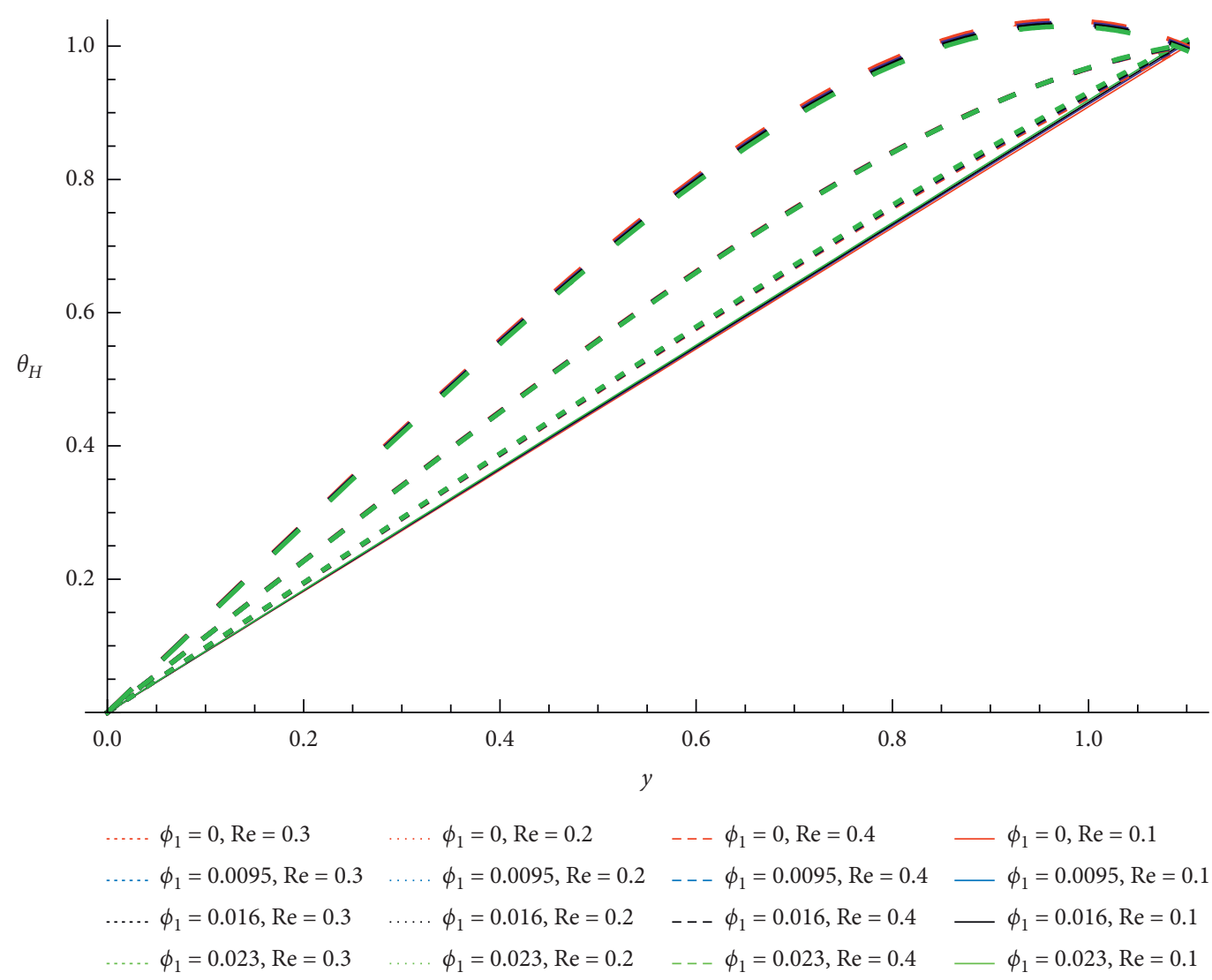

(a)

Figure 8: Continued. 


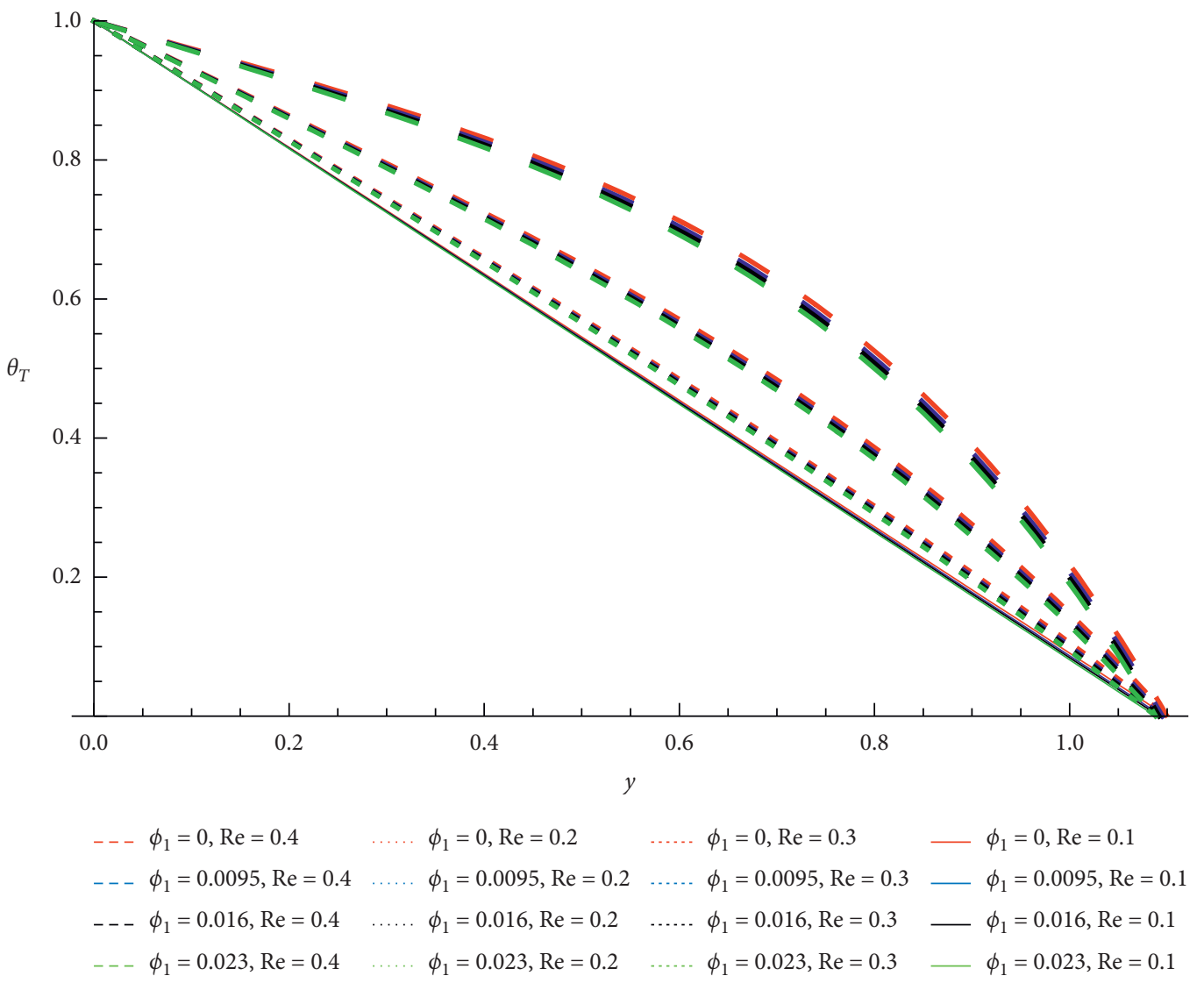

(b)

Figure 8: Temperature vs $y$ for different values of $\phi_{1}$, Re at $K=0.0005, t=0.2, \lambda_{1}=0.00011, x=0.2, \phi=0.8$, $\mathrm{Fr}=0.1, \mathrm{Q}=0.3, \alpha=(\pi / 4) \operatorname{Pr}=1, \mathrm{Ec}_{H}=1$, and $\mathrm{Ec}_{T}=1$ : (a) uniform heat flux condition; (b) uniform surface temperature.

TABLE 2: Temperature distribution at different values of Re and surface roughness parameter.

\begin{tabular}{|c|c|c|c|c|c|c|c|c|}
\hline \multirow[b]{3}{*}{$y$} & \multicolumn{4}{|c|}{$\theta_{T}$} & \multicolumn{4}{|c|}{$\theta_{H}$} \\
\hline & \multicolumn{4}{|c|}{$\mathrm{Re}=0.1$} & \multicolumn{4}{|c|}{$\mathrm{Re}=0.1$} \\
\hline & $\phi_{1}=0$ & $\phi_{1}=0.0095$ & $\phi_{1}=0.016$ & $\phi_{1}=0.023$ & $\phi_{1}=0$ & $\phi_{1}=0.0095$ & $\phi_{1}=0.016$ & $\phi_{1}=0.023$ \\
\hline 0 & 1 & 1 & 1 & 1 & 0 & 0 & 0 & 0 \\
\hline 0.2 & 0.8182 & 0.8174 & 0.8168 & 0.8162 & 0.1818 & 0.1826 & 0.1831 & 0.1837 \\
\hline 0.4 & 0.6364 & 0.6348 & 0.6337 & 0.6325 & 0.3636 & 0.3652 & 0.3663 & 0.3674 \\
\hline 0.6 & 0.4545 & 0.4522 & 0.4505 & 0.4487 & 0.5455 & 0.5478 & 0.5494 & 0.5512 \\
\hline 0.8 & 0.2727 & 0.2695 & 0.2674 & 0.2650 & 0.7273 & 0.7304 & 0.7326 & 0.7449 \\
\hline \multirow[t]{2}{*}{1.0} & 0.0909 & 0.0869 & 0.0842 & 0.0812 & 0.9091 & 0.9130 & 0.9157 & 0.9187 \\
\hline & \multicolumn{4}{|c|}{$\operatorname{Re}=0.2$} & \multicolumn{4}{|c|}{$\mathrm{Re}=0.2$} \\
\hline 0 & 1 & 1 & 1 & 1 & 0 & 0 & 0 & 0 \\
\hline 0.2 & 0.8301 & 0.8289 & 0.8281 & 0.8272 & 0.1938 & 0.1941 & 0.1944 & 0.1947 \\
\hline 0.4 & 0.6593 & 0.6569 & 0.6552 & 0.6534 & 0.3866 & 0.3873 & 0.3878 & 0.3884 \\
\hline 0.6 & 0.4849 & 0.4813 & 0.4789 & 0.4763 & 0.5758 & 0.5770 & 0.5778 & 0.5787 \\
\hline 0.8 & 0.3024 & 0.2979 & 0.2949 & 0.2916 & 0.7570 & 0.7588 & 0.7601 & 0.7615 \\
\hline \multirow[t]{2}{*}{1.0} & 0.1059 & 0.1008 & 0.0973 & 0.0936 & 0.9241 & 0.9269 & 0.9289 & 0.9310 \\
\hline & \multicolumn{4}{|c|}{$\mathrm{Re}=0.3$} & \multicolumn{4}{|c|}{$\mathrm{Re}=0.3$} \\
\hline 0 & 1 & 1 & 1 & 1 & 0 & 0 & 0 & 0 \\
\hline 0.2 & 0.8642 & 0.8622 & 0.8609 & 0.8595 & 0.2278 & 0.2275 & 0.2272 & 0.2270 \\
\hline 0.4 & 0.7245 & 0.7207 & 0.7181 & 0.7152 & 0.4518 & 0.4511 & 0.4507 & 0.4502 \\
\hline 0.6 & 0.5709 & 0.5654 & 0.5616 & 0.5575 & 0.6618 & 0.6610 & 0.6605 & 0.6599 \\
\hline 0.8 & 0.3867 & 0.3798 & 0.3751 & 0.3700 & 0.8413 & 0.8407 & 0.8403 & 0.8400 \\
\hline 1.0 & 0.1485 & 0.1409 & 0.1357 & 0.1301 & 0.9666 & 0.9669 & 0.9672 & 0.9676 \\
\hline
\end{tabular}


TABle 2: Continued.

\begin{tabular}{|c|c|c|c|c|c|c|c|c|}
\hline \multirow[b]{3}{*}{0} & \multicolumn{4}{|c|}{$\theta_{T}$} & \multicolumn{4}{|c|}{$\theta_{H}$} \\
\hline & \multicolumn{4}{|c|}{$\mathrm{Re}=0.4$} & \multicolumn{4}{|c|}{$\mathrm{Re}=0.4$} \\
\hline & 1 & 1 & 1 & 1 & 0 & 0 & 0 & 0 \\
\hline 0.2 & 0.9203 & 0.9173 & 0.9153 & 0.9132 & 0.2839 & 0.2825 & 0.2816 & 0.2807 \\
\hline 0.4 & 0.8319 & 0.8261 & 0.8222 & 0.8179 & 0.5592 & 0.5566 & 0.5448 & 0.5529 \\
\hline 0.6 & 0.7127 & 0.7043 & 0.6946 & 0.6924 & 0.8036 & 0.7999 & 0.7975 & 0.7948 \\
\hline 0.8 & 0.5256 & 0.5151 & 0.508 & 0.5003 & 0.9801 & 0.9760 & 0.9732 & 0.9702 \\
\hline 1.0 & 0.2186 & 0.2070 & 0.1992 & 0.1908 & 1.0368 & 1.0331 & 1.0307 & 1.0282 \\
\hline
\end{tabular}

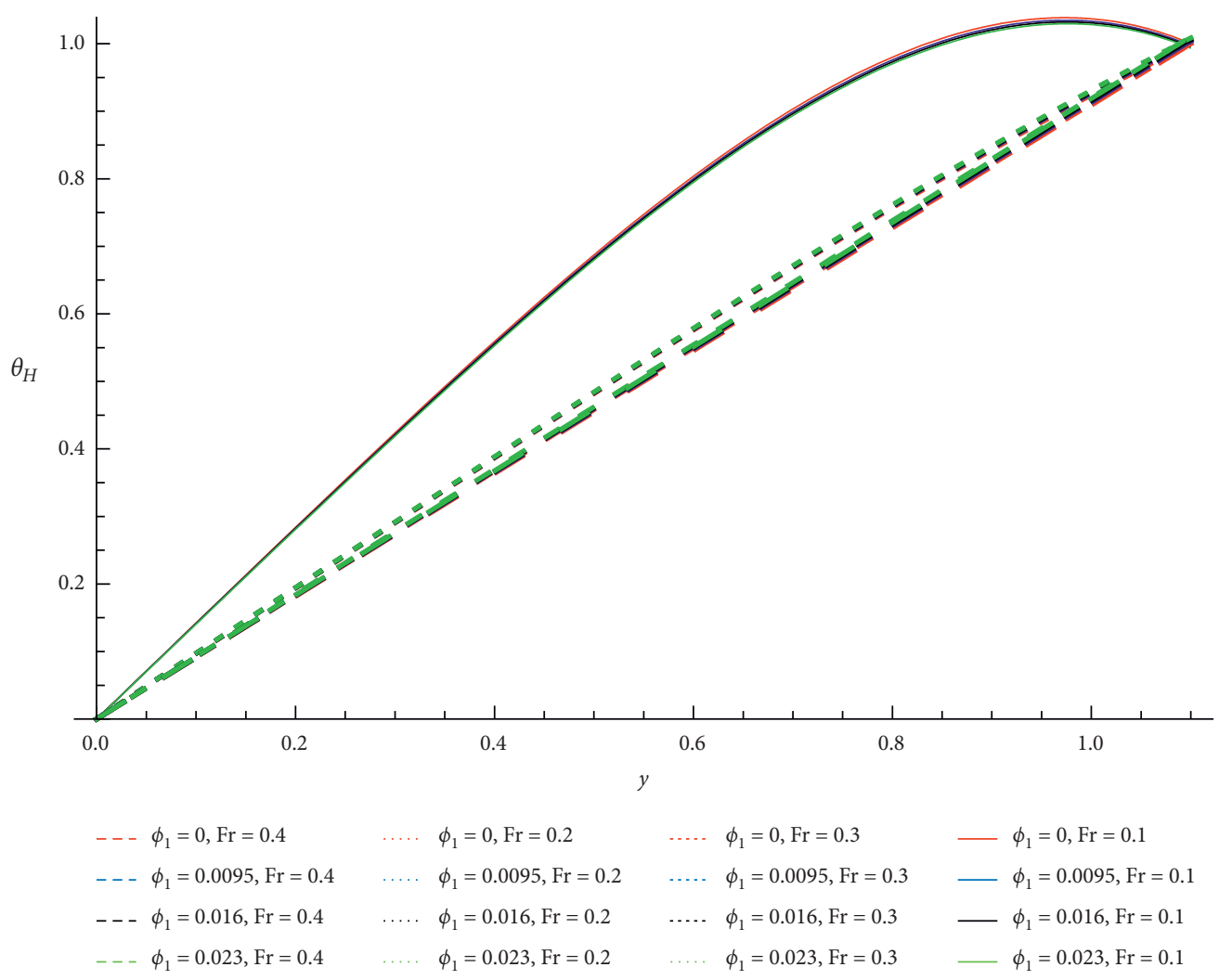

(a)

Figure 9: Continued. 


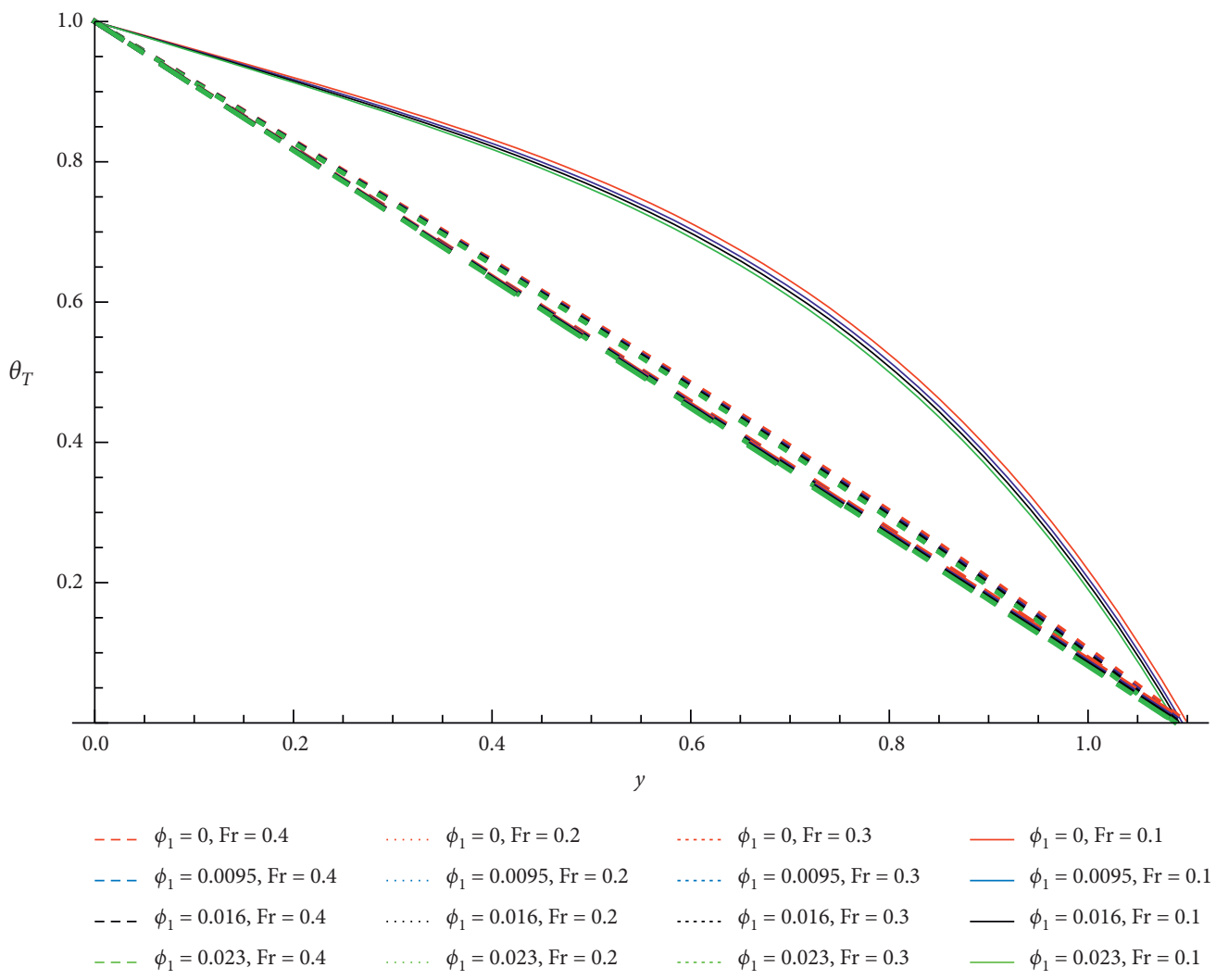

(b)

Figure 9: Temperature vs $y$ for different values of $\phi_{1}, \mathrm{Fr}$ at $K=0.0005, t=0.2, \lambda_{1}=0.00011, x=0.2, \phi=0.8, \operatorname{Re}=0.4$, $\mathrm{Q}=0.3, \alpha=(\pi / 4) \operatorname{Pr}=1, \mathrm{Ec}_{H}=1$, and $\mathrm{Ec}_{T}=1$ : (a) uniform heat flux condition; (b) uniform surface temperature.

TABLe 3: Temperature distribution at different values of Fr and surface roughness parameter.

\begin{tabular}{|c|c|c|c|c|c|c|c|c|}
\hline & \multicolumn{4}{|c|}{$\theta_{T}$} & \multicolumn{4}{|c|}{$\theta_{H}$} \\
\hline & \multicolumn{4}{|c|}{$\mathrm{Fr}=0.1$} & \multicolumn{4}{|c|}{$\mathrm{Fr}=0.1$} \\
\hline$y$ & $\phi_{1}=0$ & $\phi_{1}=0.0095$ & $\phi_{1}=0.016$ & $\phi_{1}=0.023$ & $\phi_{1}=0$ & $\phi_{1}=0.0095$ & $\phi_{1}=0.016$ & $\phi_{1}=0.023$ \\
\hline 0 & 1 & 1 & 1 & 1 & 0 & 0 & 0 & 0 \\
\hline 0.2 & 0.9203 & 0.9173 & 0.9153 & 0.9132 & 0.2839 & 0.2825 & 0.2816 & 0.2807 \\
\hline 0.4 & 0.8319 & 0.8261 & 0.8222 & 0.8179 & 0.5592 & 0.5566 & 0.5548 & 0.5529 \\
\hline 0.6 & 0.7127 & 0.7043 & 0.6986 & 0.6924 & 0.8036 & 0.7999 & 0.7975 & 0.7948 \\
\hline 0.8 & 0.5256 & 0.5151 & 0.508 & 0.5003 & 0.9801 & 0.9760 & 0.9732 & 0.9702 \\
\hline \multirow[t]{2}{*}{1.0} & 0.2186 & 0.2070 & 0.1992 & 0.1908 & 1.0368 & 1.0331 & 1.0307 & 1.0282 \\
\hline & \multicolumn{4}{|c|}{$\mathrm{Fr}=0.2$} & \multicolumn{4}{|c|}{$\mathrm{Fr}=0.2$} \\
\hline 0 & 1 & 1 & 1 & 1 & 0 & 0 & 0 & 0 \\
\hline 0.2 & 0.8301 & 0.8289 & 0.8281 & 0.8272 & 0.1938 & 0.1941 & 0.1944 & 0.1947 \\
\hline 0.4 & 0.6593 & 0.6569 & 0.6552 & 0.6534 & 0.3866 & 0.3873 & 0.3878 & 0.3884 \\
\hline 0.6 & 0.4849 & 0.4813 & 0.4789 & 0.4763 & 0.5758 & 0.5770 & 0.5778 & 0.5787 \\
\hline 0.8 & 0.3024 & 0.2979 & 0.2949 & 0.2916 & 0.7570 & 0.7588 & 0.7601 & 0.7615 \\
\hline \multirow[t]{2}{*}{1.0} & 0.1059 & 0.1008 & 0.0973 & 0.0936 & 0.9241 & 0.9269 & 0.9289 & 0.9310 \\
\hline & \multicolumn{4}{|c|}{$\mathrm{Fr}=0.3$} & \multicolumn{4}{|c|}{$\mathrm{Fr}=0.3$} \\
\hline 0 & 1 & 1 & 1 & 1 & 0 & 0 & 0 & 0 \\
\hline 0.2 & 0.8197 & 0.8188 & 0.8182 & 0.8175 & 0.1833 & 0.1840 & 0.1845 & 0.1850 \\
\hline 0.4 & 0.6393 & 0.6375 & 0.6363 & 0.6349 & 0.3666 & 0.3679 & 0.3689 & 0.3699 \\
\hline 0.6 & 0.4585 & 0.4558 & 0.4539 & 0.4519 & 0.5494 & 0.5514 & 0.5528 & 0.5544 \\
\hline 0.8 & 0.2766 & 0.2731 & 0.2707 & 0.2681 & 0.7311 & 0.7339 & 0.7359 & 0.7380 \\
\hline 1.0 & 0.0928 & 0.0886 & 0.0858 & 0.0827 & 0.9110 & 0.9147 & 0.9173 & 0.9201 \\
\hline
\end{tabular}


TABLE 3: Continued.

\begin{tabular}{|c|c|c|c|c|c|c|c|c|}
\hline \multirow[b]{3}{*}{0} & \multicolumn{4}{|c|}{$\theta_{T}$} & \multicolumn{4}{|c|}{$\theta_{H}$} \\
\hline & \multicolumn{4}{|c|}{$\mathrm{Fr}=0.4$} & \multicolumn{4}{|c|}{$\mathrm{Fr}=0.4$} \\
\hline & 1 & 1 & 1 & 1 & 0 & 0 & 0 & 0 \\
\hline 0.2 & 0.8182 & 0.8174 & 0.8168 & 0.8162 & 0.1818 & 0.1826 & 0.1831 & 0.1837 \\
\hline 0.4 & 0.6364 & 0.6348 & 0.6337 & 0.6325 & 0.3636 & 0.3652 & 0.3663 & 0.3674 \\
\hline 0.6 & 0.4545 & 0.4522 & 0.4505 & 0.4487 & 0.5455 & 0.5478 & 0.5494 & 0.5512 \\
\hline 0.8 & 0.2727 & 0.2695 & 0.2674 & 0.2650 & 0.7273 & 0.7304 & 0.7326 & 0.7349 \\
\hline 1.0 & 0.0909 & 0.0869 & 0.0842 & 0.0812 & 0.9091 & 0.9130 & 0.9157 & 0.9187 \\
\hline
\end{tabular}

at $\mathrm{Re}=0.4$ depicts a similar nature to that observed for $\operatorname{Re}=0.3$. For all values of $\operatorname{Re}$ at any given value of surface roughness parameter, as $y$ increases, $\theta_{H}$ increases while $\theta_{T}$ decreases.

From Table 3 it is found that, except at $\mathrm{Fr}=0.1$ for all other values of Fr, for any particular value of $y, \theta_{H}$ increases and $\theta_{T}$ decreases upon incrementing the value of surface roughness parameter. At $\mathrm{Fr}=0.1$, for any particular value of $y$, with an increase in surface roughness, both $\theta_{H}$ and $\theta_{T}$ decrease. For a particular choice of surface roughness parameter, as $y$ increases, $\theta_{H}$ increases and $\theta_{T}$ decreases for all values of Fr.

It can be seen that temperature profiles show a reverse behaviour at uniform surface temperature and uniform heat flux condition. For better understanding, the effect of surface roughness on temperature for various values of channel inclination Re and Fr is shown below in the tabular form.

\section{Conclusion}

The objective of this analysis is to explain the variation occurring in heat transfer due to the inner wall roughness of a nonlinear inclined channel, for transportation of Newtonian Fluid through the peristaltic mechanism. From the literature survey conducted during the study, it has been found that, so far, no attempt has been made by researchers to address the above problem. The mathematical results obtained in the present study may apply to the transportation of liquids used in various industries. This study may be useful for analysis of heat conduction in many body organs in which physiological fluid transported. The conclusion drawn from the analysis is as follows:

(1) An increase in the maximum magnitude of pressure rise is observed when the surface roughness parameter increased.

(2) The effect of surface roughness on pressure rise is majorly seen at the time $t \approx 0.3$, which corresponds to the point where maximum occlusion occurs.

(3) The maximum pressure rise value increases, as the angle of inclination is increased from 0 to $(\pi / 2)$.

(4) Pressure rise increases at all points for the period of a wave as the inclination of the channel is increased from 0 to $(\pi / 2)$.

(5) For a particular choice of pressure rise value, the flow rate increases with angle of inclination from 0 to $(\pi / 2)$.
(6) The significant effect of the angle of the inclination of the channel has been observed in the region $0.6<\Phi<0.94$.

(7) Maximum flow rate increases with an inclination of the channel from 0 to $(\pi / 2)$.

(8) The value of maximum pressure rise and flow rate rises when Reynolds number is augmented.

(9) For any value of Reynolds number, the maximum pressure attains higher values when the surface roughness parameter is increased.

(10) The maximum pressure rise and maximum flow rate decrease as the Froude number is increased.

(11) For any particular Froude number, maximum pressure rise attains a higher magnitude at a higher surface roughness parameter.

(12) The temperature profiles show opposite behaviours at uniform heat flux conditions to that of uniform surface temperature conditions.

(13) Excluding the value of $\theta_{H}$ at zero inclination, the value of $\theta_{H}$ and $\theta_{T}$ decreases with an increase in surface roughness, at all angles of inclination for any particular value of $y$.

\section{Nomenclature}

$H$ : Vertical displacement

$b$ : Amplitude of the wave

a: Inlet half channel width

$K$ : Nonuniformity parameter

$c$ : Wave velocity

$t$ : Time

$\lambda$ : Wavelength

$b_{1}$ : Roughness height

$\lambda_{1}:$ Pitch

$X$ : Axial variable

$Y: \quad$ Transverse variable

$U: \quad$ Axial velocity

$V: \quad$ Transverse velocity

$\rho: \quad$ Density

$p: \quad$ Pressure

$\mu$ : Coefficient of viscosity

g: Gravitational acceleration

$\alpha: \quad$ Angle of inclination of channel

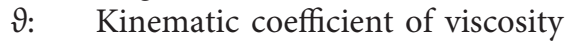

$c_{p}$ : Specific heat at constant pressure

$k$ : Thermal conductivity 
T: $\quad$ Temperature of fluid

$T_{w}$ : Temperature at the wall

$T_{c}$ : Temperature at centre of the channel

$\theta_{H}$ : Dimensionless temperature in case of uniform heat flux

$\theta_{T}$ : Dimensionless temperature in case of uniform surface temperature

$\mathrm{Ec}_{H}$ : Eckert number in case of uniform heat flux

$\mathrm{Ec}_{T}$ : Eckert number in case of uniform surface temperature

Pr: Prandtl number

$h$ : Dimensionless vertical displacement

$t^{\prime}$ : Dimensionless time

$\phi_{1}$ : Roughness parameter

$\phi: \quad$ Amplitude ratio

$X^{\prime}$ : Dimensionless axial variable

$Y^{\prime}$ : Dimensionless transverse variable

$U^{\prime}$ : Dimensionless axial velocity

$V^{\prime}$ : Dimensionless transverse velocity

$p^{\prime}$ : Dimensionless pressure

Re: Reynolds number

Fr: Froude number

$\delta$ : Wavenumber

$\Delta p:$ Pressure rise

$F$ : $\quad$ Friction force at the wall of channel

L: $\quad$ Length of channel

q: Instantaneous flow rate

Q: $\quad$ Time mean flow rate.

\section{Data Availability}

The data used to support the findings of this study are available from the first author upon request.

\section{Conflicts of Interest}

The authors declare that they have no conflicts of interest.

\section{References}

[1] T. W. Latham, "Fluid motion in a peristaltic pump," M.S. thesis, Massachusetts Institute of Technology, Cambridge, MA, USA, 1966.

[2] A. H. Shapiro, M. Y. Jaffrin, and S. L. Weinberg, "Peristaltic pumping with long wavelengths at low Reynolds number," Journal of Fluid Mechanics, vol. 37, no. 4, pp. 799-825, 1969.

[3] B. B. Gupta and V. Seshadri, "Peristaltic pumping in nonuniform tubes," Journal of Biomechanics, vol. 9, no. 2, pp. 105-109, 1976.

[4] G. Radhakrishnamacharya and V. R. Murty, "Heat transfer to peristaltic in a non uniform channel," Defence Science Journal, vol. 43, no. 3, pp. 275-280, 1993.

[5] S. R. El Koumy, E. S. I. Barakat, and S. I. Abdelsalam, "Hall and porous boundaries effects on peristaltic transport through porous medium of a Maxwell model," Transport in Porous Media, vol. 94, no. 3, pp. 643-658, 2012.

[6] K. S. Mekheimer, S. R. Komy, and S. I. Abdelsalam, "Simultaneous effects of magnetic field and space porosity on compressible Maxwell fluid transport induced by a surface acoustic wave in a microchannel," Chinese Physics B, vol. 22, no. 12, Article ID 124702, 2013.
[7] Y. Abd Elmaboud, S. I. Abdelsalam, and K. S. Mekheimer, "Couple stress fluid flow in a rotating channel with peristalsis," Journal of Hydrodynamics, vol. 30, no. 2, pp. 307-316, 2018.

[8] D. Tripathi and O. Anwar Bég, "Peristaltic propulsion of generalized burgers' fluids through a non-uniform porous medium: a study of chyme dynamics through the diseased intestine," Mathematical Biosciences, vol. 248, pp. 67-77, 2014.

[9] S. I. Abdelsalam and K. Vafai, "Combined effects of magnetic field and rheological properties on the peristaltic flow of a compressible fluid in a microfluidic channel," European Journal of Mechanics-B/Fluids, vol. 65, pp. 398-411, 2017.

[10] S. I. Abdelsalam and M. M. Bhatti, "The study of nonNewtonian nanofluid with hall and ion slip effects on peristaltically induced motion in a non-uniform channel," RSC Advances, vol. 8, no. 15, pp. 7904-7915, 2018.

[11] I. M. Eldesoky, S. I. Abdelsalam, W. A. El-Askary, and M. M. Ahmed, "Concurrent development of thermal energy with magnetic field on a particle-fluid suspension through a porous conduit," BioNanoScience, vol. 9, no. 1, pp. 186-202, 2019.

[12] S. A. Victor and V. L. Shah, "Heat transfer to blood flowing in a tube," Biorheology, vol. 12, no. 6, pp. 361-368, 1975.

[13] S. A. Victor and V. L. Shah, "Steady state heat transfer to blood flowing in the entrance region of a tube," International Journal of Heat and Mass Transfer, vol. 19, no. 7, pp. 777-783, 1976.

[14] L. M. Srivastava and V. P. Srivastava, "Peristaltic transport of blood: casson model-II," Journal of Biomechanics, vol. 17, no. 11, pp. 821-829, 1984.

[15] K. S. Mekheimer, "Peristaltic flow of blood under effect of a magnetic field in a non-uniform channels," Applied Mathematics and Computation, vol. 153, no. 3, pp. 763-777, 2004.

[16] S. I. Abdelsalam and K. Vafai, "Particulate suspension effect on peristaltically induced unsteady pulsatile flow in a narrow artery: blood flow model," Mathematical Biosciences, vol. 283, pp. 91-105, 2017.

[17] Y. Abd Elmaboud, S. I. Abdelsalam, K. S. Mekheimer, and K. Vafai, "Electromagnetic flow for two-layer immiscible fluids," Engineering Science and Technology, an International Journal, vol. 22, no. 1, pp. 237-248, 2019.

[18] S. I. Abdelsalam, M. M. Bhatti, A. Zeeshan, A. Riaz, and O. A. Bég, "Metachronal propulsion of a magnetised particlefluid suspension in a ciliated channel with heat and mass transfer," Physica Scripta, vol. 94, no. 11, p. 115301, 2019.

[19] M. Sohail, R. Naz, and S. I. Abdelsalam, "On the onset of entropy generation for a nanofluid with thermal radiation and gyrotactic microorganisms through 3D flows," Physica Scripta, vol. 95, no. 4, Article ID 045206, 2020.

[20] S. I. Abdelsalam and M. Sohail, "Numerical approach of variable thermophysical features of dissipated viscous nanofluid comprising gyrotactic micro-organisms," Pramana, vol. 94, no. 1, p. 67, 2020.

[21] M. Sohail, R. Naz, and S. I. Abdelsalam, “Application of nonFourier double diffusions theories to the boundary-layer flow of a yield stress exhibiting fluid model," Physica A: Statistical Mechanics and Its Applications, vol. 537, Article ID 122753, 2020.

[22] H. Sadaf and S. I. Abdelsalam, "Adverse effects of a hybrid nanofluid in a wavy non-uniform annulus with convective boundary conditions," RSC Advances, vol. 10, no. 26, pp. 15035-15043, 2020. 
[23] S. I. Abdelsalam and M. M. Bhatti, "The impact of impinging $\mathrm{TiO}_{2}$ nanoparticles in Prandtl nanofluid along with endoscopic and variable magnetic field effects on peristaltic blood flow," Multidiscipline Modeling in Materials and Structures, vol. 14, no. 3, pp. 530-548, 2018.

[24] S. I. Abdelsalam and M. M. Bhatti, "New insight into AuNP applications in tumour treatment and cosmetics through wavy annuli at the nanoscale," Scientific Reports, vol. 9, p. 260, 2019.

[25] S. I. Abdelsalam and M. M. Bhatti, "Anomalous reactivity of thermo-bioconvective nanofluid towards oxytactic microorganisms," Applied Mathematics and Mechanics, vol. 41, no. 5, pp. 711-724, 2020.

[26] I. M. Eldesoky, S. I. Abdelsalam, W. A. El-Askary, A. M. ElRefaey, and M. M. Ahmed, "Joint effect of magnetic field and heat transfer on particulate fluid suspension in a catheterized wavy tube," BioNanoScience, vol. 9, pp. 723-739, 2019.

[27] S. W. Park, M. Intaglietta, and D. M. Tartakovsky, "Impact of endothelium roughness on blood flow," Journal of Theoretical Biology, vol. 300, pp. 152-160, 2012.

[28] H. E. Burton and D. M. Espino, "The effect of mechanical overloading on surface roughness of the coronary arteries," Applied Bionics and Biomechanics, vol. 2019, Article ID 2784172, 8 pages, 2019.

[29] M. M. Bhatti, M. Marin, A. Zeeshan, R. Ellahi, and S. I. Abdelsalam, "Swimming of motile gyrotactic microorganisms and nanoparticles in blood flow through anisotropically tapered arteries," Frontiers in Physics, vol. 8, 2020.

[30] R. W. Veatch, The Effect of Surface Roughness on Fluid Flow through Narrow Rectangular Passages, Society of Petroleum Engineers, Richardson, TX, USA, 1965.

[31] J. C. Han, Y. M. Zhang, and C. P. Lee, "Augmented heat transfer in square channels with parallel, crossed, and V-shaped angled ribs," Journal of Heat Transfer, vol. 113, no. 3, pp. 590-596, 1991.

[32] M. E. Taslim, T. Li, and D. M. Kercher, "Experimental heat transfer and friction in channels roughened with angled, V-shaped and discrete ribs on two opposite walls," in Proceedings of the International Gas Turbine and Aeroengine Congress and Exposition, pp. 13-16, Hague, Netherlands, June 1994.

[33] J. B. Taylor, A. L. Carrano, and S. G. Kandlikar, "Characterization of the effect of surface roughness and texture on fluid flow-past, present, and future," International Journal of Thermal Sciences, vol. 45, no. 10, pp. 962-968, 2006.

[34] R. N. Wagner and S. G. Kandlikar, "Effects of structured roughness on fluid flow at the microscale level," Heat Transfer Engineering, vol. 33, no. 6, pp. 483-493, 2012.

[35] V. V. Dharaiya and S. G. Kandlikar, "A numerical study on the effects of 2D structured sinusoidal elements on fluid flow and heat transfer at microscale," International Journal of Heat and Mass Transfer, vol. 57, no. 1, pp. 190-201, 2013.

[36] M. Kharati-Koopaee and M. Zare, "Effect of aligned and offset roughness patterns on the fluid flow and heat transfer within microchannels consist of sinusoidal structured roughness," International Journal of Thermal Sciences, vol. 90, pp. 9-23, 2015.

[37] M. Attalla, H. M. Maghrabie, and E. Specht, “An experimental investigation on fluid flow and heat transfer of rough minichannel with rectangular cross section," Experimental Thermal and Fluid Science, vol. 75, pp. 199-210, 2016. 